\title{
Ausgezeichnete Innovationen
}

Im Rahmen einer Gala in Berlin ist der von Springer Medizin gestiftete

Galenus-von-Pergamon-Preis für pharmazeutische Innovationen verliehen worden.

Mit dem CharityAward wurde auch ehrenamtliches Engagement gewürdigt.

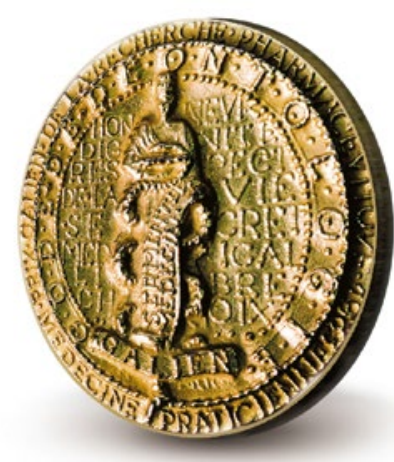

Mit dem Galenus-Preis zeichnet Springer Medizin herausragende Leistungen in der Pharmakologie aus. Schirmherrin ist die Bundesforschungsministerin Prof. Johanna Wanka. Stellvertretend überreichte der Parlamentarische Staatssekretär im Bundesministerium für Bildung und Forschung Dr. Georg Schütte die Preise auf einer Gala im Berliner Kongresszentrum Axica. Die Preisträger waren am Nachmittag zuvor in geheimer Abstimmung durch 14 Experten und unter dem Juryvorsitz von Prof. Erland Erdmann, Köln, ermittelt worden.

\section{Grundlagenforschung}

In der Kategorie „Pharmakologische Grundlagenforschung“ nahm die Forschergruppe um Prof. Florian Bassermann von der TU München die Auszeichnung entgegen. Die Wissenschaftler der Abteilung Hämatologische/Internistische Onkologie der III. Medizinischen Klinik und Poliklinik beschrieben erstmals den molekularen Mechanismus, der für die Antitumorwirkung immunmodulierender Substanzen (IMiDs) verantwortlich ist. Sie fanden heraus, dass die Arzneimittel Krebszellen schädigen, indem sie einen Komplex der Zelleiweiße CD147 und MCT1 destabilisieren. Diese grundlegende Entdeckung kann in Zukunft den Weg zu neuen Medikamenten in der Krebstherapie ebnen.

\section{Primary Care}

Den Galenus-Preis in der Kategorie „Primary Care“ erhielt das Medikament Entresto ${ }^{\circledR}$ von Novartis. Die Fixkombination von Sacubitril und Valsartan ist der erste verfügbare Angiotensinrezeptor-Neprilysin-Inhibitor (ARNI). Er kann bei Patienten mit symptomatischer chronischer Herzinsuffizienz und reduzierter Ejektionsfraktion das Überleben verlängern.

\section{Specialist Care}

In der Kategorie „Specialist Care“ vergab die Jury den Preis in diesem Jahr an Cinqaero ${ }^{\star}$. Das Arzneimittel von Teva enthält Reslizumab, einen Antikörper gegen Interleukin-5 (IL-5). Er reduziert die Zahl eosinophiler Granulozyten und führt so bei $\mathrm{Pa}$ tienten mit schwerem eosinophilem Asthma bronchiale zur Verringerung der Exazerbationsfrequenz sowie zur Verbesserung von Lungenfunktion und Lebensqualität.

\section{Orphan Drugs}

Die Kategorie „Orphan Drugs“ wurde für Innovationen für Patienten mit einer seltenen Krankheit geschaffen. Diesjähriger Gewinner ist Venclyxto (Venetoclax) von AbbVie. Venetoclax ist der erste orale Inhibitor des in Zellen von Patienten mit chronischer lymphatischer Leukämie (CLL) überexprimierten B-ZellLymphom-2-Proteins (BCL-2). BCL-2 hemmt den intrinsischen mitochondrialen Signalweg des programmierten Zelltods (Apoptose). Durch die Blockade von BCL-2 wird die Fähigkeit der Tumorzelle zur Apoptose wiederhergestellt. Zugelassen ist Venclyxto ${ }^{\star}$ für Hochrisikopatienten mit CLL. Auch Patienten, die auf Chemoimmuntherapie und/oder einen Inhibitor des B-Zell-Rezeptor-Signalwegs nicht ausreichend ansprechen, profitieren. Bisherige Studien zur Wirksamkeit und Verträglichkeit von Venetoclax zeigten hohe Ansprechraten, eine rasch einsetzende Wirkung und ein gut handhabbares Nebenwirkungsprofil. red.

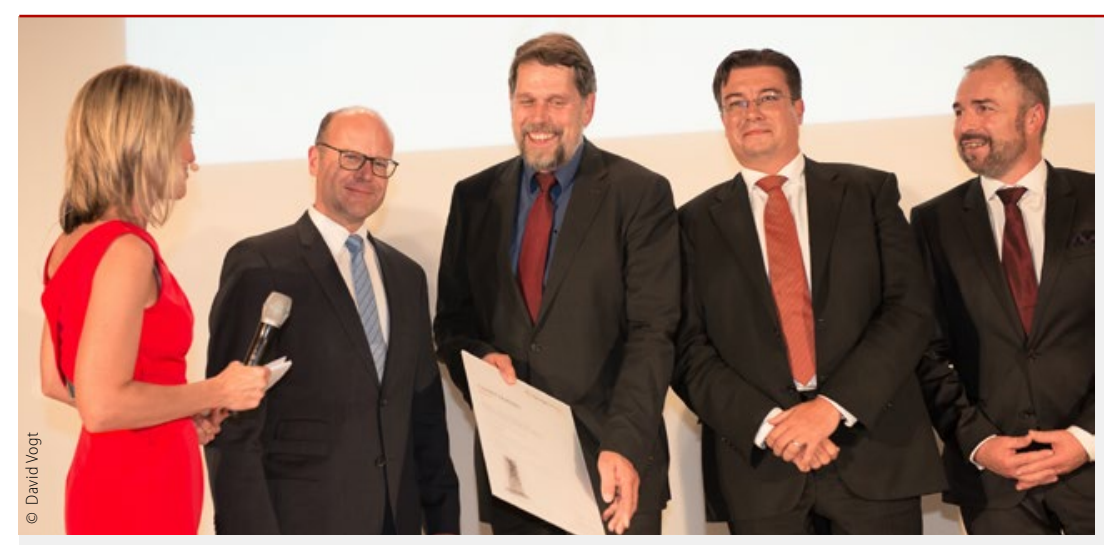

Prof. Mathias Freund (3.v. l.) freut sich über den 3. Platz beim Springer Medizin CharityAward 2017. Mit im Bild (v. l.) Moderatorin Yve Fehring, BMG-Vertreter Oliver Schenk, Sponsoren Dr. Christian Rybak (Ehlers, Ehlers \& Partner) und Dr. Nicolai Kohlschmidt (Berufsverband Dt. Humangenetiker).

\section{CharityAward 2017}

Mit dem CharityAward zeichnet Springer Medizin unter der Schirmherrschaft von Bundesgesundheitsminister Hermann Gröhe ehrenamtliches Engagement im Gesundheitswesen aus.

Platz 1 belegte das Präventionsprojekt "Streetworkt" der Off Road Kids Stiftung und Bahn-BKK. Das Projekt klärt junge, auf der Straße lebende Menschen über Gesundheitsrisiken auf.

Platz 2 ging an die Münchner Studenteninitiative AIAS, die über Blutkrebs aufklärt und eine Registrierung zum Stammzellspender direkt am Campus ermöglicht.

Mit Platz 3 ausgezeichnet wurde die Deutsche Stiftung für junge Erwachsene mit Krebs. Ihr Projekt "Junges Krebsportal“ (https://www.junges-krebsportal.de) unterstützt Menschen, die im Alter zwischen 18 und 39 Jahren an Krebs erkranken. 